\title{
Correction to: Laser lithotripsy for proximal ureteral calculi in adults: can 3D CT texture analysis help predict treatment success?
}

\author{
Rui Wang ${ }^{1} \cdot$ Yunshan $\mathrm{Su}^{2}$ (D) Chongwen $\mathrm{Mao}^{2} \cdot$ Song $\mathrm{Li}^{3} \cdot$ Mengjing You $^{2} \cdot$ Shutian Xiang ${ }^{2}$ \\ Published online: 22 June 2021 \\ (C) European Society of Radiology 2021
}

\section{Correction to: European Radiology (2021) 31:3734-3744 https://doi.org/10.1007/s00330-020-07498-x}

Following the request of the Editors, the authors wish to clarify the following points concerning the original article "Laser lithotripsy for proximal ureteral calculi in adults: can 3D CT texture analysis help predict treatment success?" (Wang et al., https://doi.org/10.1007/s00330-020-07498-x) published online first on November 19, 2020:

1. The patient cohorts in the European Radiology publication have a partial overlap with the Chinese language article "Estimate therapeutic effect of laser lithotripsy with CT three-dimensional texture analysis in patients with ureteral calculi (Wang et al, DOI:https://doi.org/10. 19300/j.2020.L17765, published in Int J Med Radiol)". The overlap patients are 21 with proximal ureteral calculi diagnosed by certain $\mathrm{CT}$ scanner.
The online version of the original article can be found at https://doi.org/ 10.1007/s00330-020-07498-x

\section{Yunshan $\mathrm{Su}$}

13608859802@139.com

1 The Clinical School of Medicine, Dali University, 2 Shenghong Road, Gucheng, Dali 671000, Yunnan Province, China

2 Department of Radiology, Second People's Hospital of Yunnan Province, 176 Qingnian Road, Wuhua District, Kunming 650021, Yunnan Province, China

3 Department of Urology, Second People's Hospital of Yunnan Province, 176 Qingnian Road, Wuhua District, Kunming 650021, Yunnan Province, China
2. 72 patients in the Chinese language article which published earlier in Int J Med Radiol provided the calculi in all segment of the ureter images by one CT scanner from Jan 2016 to Sept 2018; while 94 patients in the article published in European Radiology provided the proximal ureteral calculi images by multiple CT scanners from Jan 2016 to March 2019.

3. In addition, the inclusion criteria, grouping conditions of qualified cases and statistical methods in the two publications were totally different.

Publisher's note Springer Nature remains neutral with regard to jurisdictional claims in published maps and institutional affiliations. 